\title{
Comparação das inclinações lateral e anteroposterior no equilíbrio estático entre jovens, adultos e idosos
}

\author{
Comparison of lateral and anteroposterior slope in static balance among young, adults and elderly
}

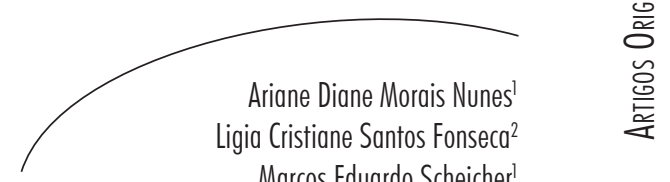

Resumo

Introdução: Com o aumento da população idosa, fica evidente a importância de compreender e explorar o processo de envelhecimento humano, o qual acarreta transformações morfológicas e funcionais. Objetivos: Avaliar e comparar as inclinações anteroposterior e lateral do centro de gravidade por faixas etárias. Métodos: Estudo transversal e exploratório. Fizeram parte da pesquisa 135 sujeitos, separados em faixas etárias: Grupo 1: 20-29 anos ( $\mathrm{n}=26$ ), Grupo 2: 30-39 anos ( $\mathrm{n}=26)$, Grupo 3: 40-49 ( $n=25)$, Grupo 4: 50-59 anos ( $n=30)$ e Grupo 5: $\geq 60$ anos ( $n=28)$. Esses sujeitos foram selecionados de acordo com o escore do Miniexame de Estado Mental e pela capacidade de permanecer em pé por, pelo menos, 90 segundos. As posturas foram avaliadas por meio do software de avaliação postural (SAPO). As comparações entre os valores de inclinações dados pelo SAPO foram feitas pelo teste de Mann-Whitney (comparação par-a-par), sendo aceito um valor de $\mathrm{p} \leq 0,05$ como significante. Resultados: Na comparação da média da assimetria frontal entre as faixas etárias, observa-se maior lateralização com o avançar da idade, com valores significativos nos grupos 50-59 e $\geq 60$ anos. Na comparação da média da assimetria sagital entre as faixas etárias, valores são significativos no grupo dos $\geq 60$ anos. Conclusões: Após avaliar e comparar as assimetrias sagitais e frontais, pode-se apontar que na medida em que a idade avança, há tendência ao aumento de suas projeções anteriores e laterais comparadas ao centro de gravidade.

\section{Abstract}

Introduction: With the increase of elderly population, it becomes important to understand and assess the process of human aging, which causes morphological and functional. Objectives: To evaluate and compare antero posterior and lateral slopes of center of gravity by age groups. Methods: Cross sectional and exploratory, including 135 subjects, divided into age groups: Group 1: 20-29 years ( $\mathrm{n}=26)$, Group 2: 30-39 years $(n=26)$, Group 3: 40-49 ( $n=25)$, Group 4: 50-59 years $(n=30)$ and Group 5: $\geq 60$ years

\footnotetext{
Departamento de Fisioterapia e Terapia Ocupacional, Faculdade de Filosofia e Ciências. Universidade Estadual Paulista. Marília, SP, Brasil.

2 Instituto de Biociências, Universidade Estadual Paulista. Rio Claro, SP, Brasil

Correspondência / Correspondence

Marcos Eduardo Scheicher

E-mail:mscheicher@marilia.unesp.br
}

Palavras-chave: Postura

Estática. Assimetria

Frontal. Assimetria Sagital.

Envelhecimento. 
$(\mathrm{n}=28)$. These subjects were selected according to the score of the Mini-mental State Examination and the ability to stand for at least 90 seconds. The attitudes were assessed using the Postural Assessment Software (SAPO). Comparisons among the values given by SAPO slopes were made using the Mann-Whitney test (comparison peer-to-peer), and accepted a value of $\mathrm{p} \leq 0.05$ as significant. Results: Comparing the average frontal asymmetry between the age groups, greater lateralization is observed with advancing age, with significant values in the groups 50-59 and $\geq 60$ years. Comparing sagittal asymmetry averages among the age groups, significant values were found in the $\geq 60$ years group. Conclusions: After evaluating and comparing the sagittal and frontal asymmetry, it may be pointed out that as age advances, there is a tendency to increase their anterior and lateral projections compared to the center of gravity.
Key words: Static Posture. Frontal Asymmetry. Sagittal Asymmetry. Aging.

\section{INTRODUÇ̃̃O}

Segundo dados do IBGE (2010), no Brasil atual a população idosa representa um percentual de $7,4 \%$, ou seja, mais de 14 milhões de pessoas. ${ }^{1}$ Diante disso, fica evidente a importância de compreender e explorar o processo de envelhecimento humano, o qual acarreta transformações morfológicas e funcionais.

Doenças associadas ao envelhecimento estão presentes em grande parte da população idosa e podem exacerbar as deteriorações sensoriais e motoras consequentes do processo natural de envelhecimento, levando essa população a ter prejuízos no controle postural. ${ }^{2}$ Este é definido como um processo pelo qual o sistema nervoso central gera padrões de atividade muscular requeridos para regular a relação entre o centro de massa corporal e a base de suporte. ${ }^{3}$

Assumindo que o controle postural possui dois objetivos comportamentais, denominados "orientação" e "equilíbrio postural", e que esses objetivos são alcançados, segundo Horak \& Macpherson, ${ }^{4}$ a partir de um intrincado e dinâmico relacionamento entre informação sensorial e atividade muscular, o desempenho do sistema de controle postural de idosos poderia ser alterado como resultado das mudanças que ocorrem com o passar dos anos nos sistemas sensoriais e motor.
Nesse contexto, o declínio desses sistemas tem sido correlacionado com o exponencial aumento do número de quedas em idosos..$^{5-7} \mathrm{~A}$ queda representa a consequência mais grave das alterações do controle postural, pois prejudica a independência do idoso, ${ }^{2}$ além de gerar elevados gastos para a saúde pública brasileira. De acordo com o Ministério da Saúde, foram gastos R \$ 81 milhões de reais com fraturas em idosos e calculados 1.478 óbitos por fraturas de fêmur no ano de $2009 .{ }^{8}$

Estudos têm apontado a deficiência do controle postural e as perturbações do equilíbrio como fatores importantes na ocorrência de quedas em idosos. ${ }^{9-11}$ Saber se há ou não uma progressão nessas alterações posturais, portanto, é de fundamental importância para que estratégias de prevenção e/ou correção de desvios posturais possam ser elaboradas, de modo a evitar o aumento acelerado da incidência de quedas na população idosa. Assim, o objetivo deste estudo foi avaliar e comparar as inclinações anteroposterior e laterais do centro de gravidade, por faixas etárias.

\section{METODOLOGIA}

Foi realizado estudo transversal e exploratório com as coletas ocorrendo de julho a dezembro de 2011. Inicialmente, foram recrutadas 150 
pessoas de forma aleatória, das quais 15 não foram incluídas por apresentarem sintomas de instabilidade (tontura ou vertigem), artrite, problemas visuais não corrigidos, hipotensão postural; por utilizarem medicamentos como sedativos, hipnóticos, ansiolíticos e antidepressivos; e por não conseguirem permanecer em pé por, pelo menos, 90 segundos. Além disso, os participantes que apresentaram estado cognitivo deficiente (pontuação $<18$ no Miniexame de Estado Mental), ${ }^{12}$ também não foram incluídos na pesquisa. Fizeram parte do estudo, então, 135 sujeitos, separados em faixas etárias: Grupo 1: 20-29 anos ( $\mathrm{n}=26)$; Grupo 2: 30-39 anos ( $\mathrm{n}=26)$; Grupo 3: 40-49 anos ( $\mathrm{n}=25)$; Grupo 4: 50-59 anos ( $\mathrm{n}=30)$; e Grupo 5: $\geq 60$ anos ( $\mathrm{n}=28$ ). O Grupo 1 foi composto por estudantes universitários. Os demais foram compostos por moradores da comunidade da cidade de Marília-SP. As faixas etárias foram determinadas a critério dos pesquisadores, com o intuito de verificar a alteração na postura estática a cada período de dez anos.

Todos os sujeitos da amostra foram submetidos a uma avaliação inicial feita pelos pesquisadores por meio de um questionário, com informações autorreferidas sobre dados pessoais; ocorrência de quedas e fraturas consequentes; utilização de medicamentos; prática de atividade física e presença de doenças. Além disso, foram avaliadas também a estatura e a massa corporal. Em relação à presença de doenças, a seguinte pergunta foi feita aos participantes: algum médico ou outro profissional da saúde lhe disse que o(a) senhor(a) tem alguma doença?

\section{Avaliação da postura estática}

As inclinações foram avaliadas por meio do programa SAPO (software de avaliação postural), que é gratuito e disponibilizado pela Fundação de Amparo à Pesquisa do Estado de São Paulo (FAPESP). Foi criado com o intuito de tornar a análise da avaliação postural mais fidedigna, com registro de fotografias do corpo inteiro do indivíduo em diferentes planos durante a postura ereta, com banco de dados, fundamentação científica e acesso integral pela internet. De acordo com as instruções e padronizações do SAPO, foram digitalizadas posições de pontos anatômicos em fotografias, por meio de uma câmera fotográfica digital (Sony Cyber-Shot) ${ }^{\circledR}$ com resolução de 7.2 mega pixels fixa em um tripé a uma altura de $0,90 \mathrm{~m}$ do chão, posicionada a três metros de distância do avaliado.

As fotos foram espacialmente calibradas de acordo com um referencial fixo constituído por um fio de prumo com a marcação de um metro. Esses pontos correspondem tipicamente a referências anatômicas sobre o corpo do sujeito, e foram delimitados por meio da anatomia palpatória e marcados com pequenas esferas de isopor fixadas sobre a pele do participante com fita adesiva dupla face, sempre pelo mesmo avaliador. A partir dos pontos digitalizados, o programa fornece automaticamente valores em forma de porcentagem das assimetrias sagital e frontal, e cria a projeção gráfica do centro de gravidade. Um estudo com o objetivo de investigar a acurácia do SAPO concluiu que o programa é uma ferramenta confiável para avaliação postural..$^{13}$ 

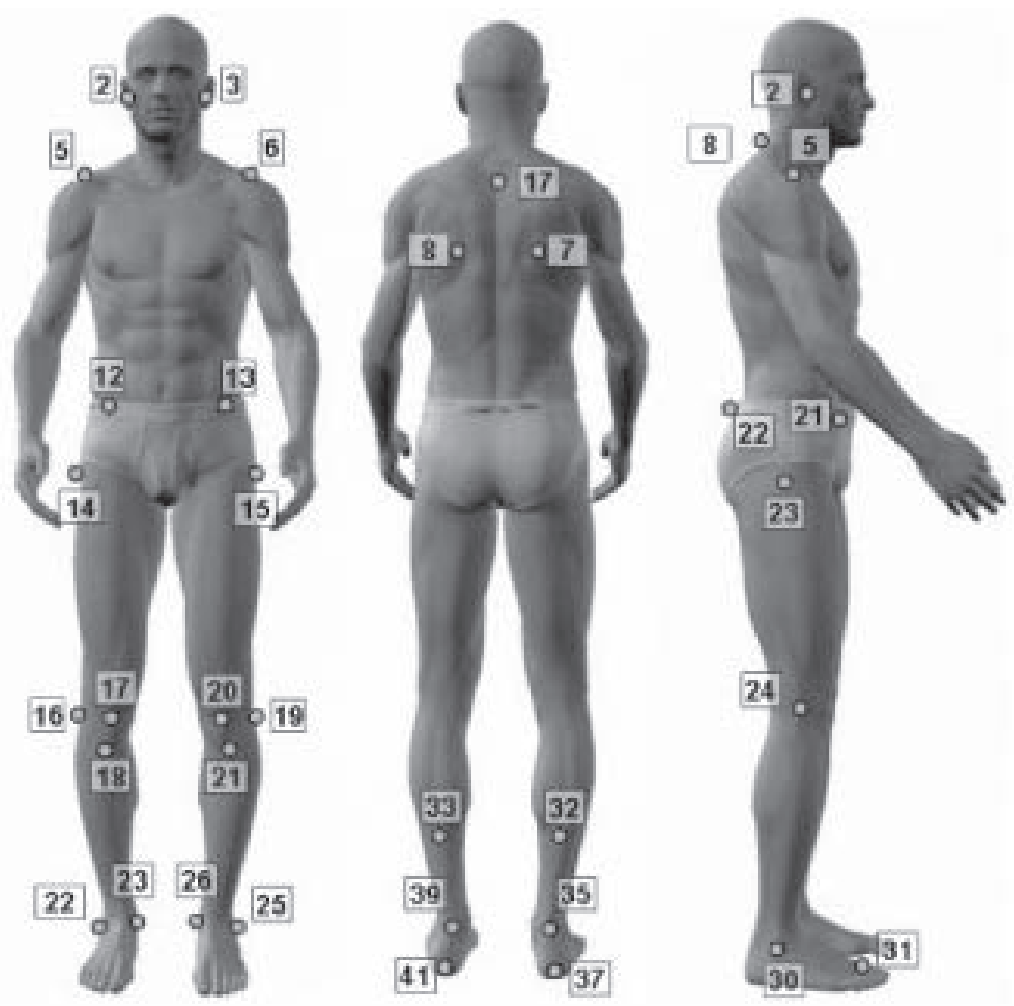

Figura 1 - Referências anatômicas do protocolo do software SAPO. Vista anterior (2,3 tragus direito e esquerdo; 5,6 acrômio direito e esquerdo; 12,13 espinha ilíaca anterossuperior direita e esquerda; 14,15 trocânter maior direito e esquerdo; 16,19 projeção lateral da linha articular do joelho direito e esquerdo; 17,20 centro da patela direita e esquerda; 18,21 tuberosidade da tíbia direita e esquerda; 22,25 maléolos laterais; 23,26 maléolos mediais). Vista posterior (7,8 ângulo inferior da escápula direita e esquerda; 17 vértebra C7;32,33 ponto medial da perna; 35,39 linha intermaleolar; 37,41 tendão calcâneo bilateralmente). Vista lateral (2 tragus; 8 vértebra C7; 5 acrômio; 21 espinha ilíaca posterossuperior; 23 trocânter maior, 24 projeção da linha articular do joelho; 30 maléolo lateral; 31 região entre o segundo e terceiro metatarso).

Fonte: http://sapo.incubadora.fapesp.br. Marília-SP, 2011.

Todos os participantes selecionados assinaram um Termo de Consentimento Livre e Esclarecido e também um Termo de Consentimento de Fotos. O trabalho foi submetido ao Comitê de Ética em Pesquisa da Faculdade de Filosofia e Ciências (FFC) da UNESP de Marília, e aprovado de acordo com o processo nº. 0102/2010.

\section{Análise estatística}

Osdados antropométricos foram apresentados como média \pm desvio-padrão. As comparações entre os valores de inclinações foram feitas pelo teste de Mann-Whitney (comparação par-a-par) e pelo teste de Kruskal-Wallis (três ou mais grupos), sendo aceito um valor de $\mathrm{p} \leq 0,05$ como significante.

\section{RESULTADOS}

A tabela 1 mostra as características dos sujeitos em relação ao número de participantes em cada faixa etária, idade e índice de massa corpórea. Em relação à média do índice de massa corpórea, observa-se diferença estatística, o que é comum com o avançar da idade. 
Tabela 1 - Características dos sujeitos avaliados. Marília-SP, 2011

\begin{tabular}{lccccc}
\hline & $20-29$ anos & $30-39$ anos & $40-49$ anos & $50-59$ anos & $\geq 60$ anos \\
\hline $\mathrm{n}$ & 26 & 26 & 25 & 30 & 28 \\
Gênero (m/f) & $13 / 13$ & $9 / 17$ & $4 / 21$ & $7 / 23$ & $13 / 15$ \\
Média de idade \pm dp & $21,3 \pm 1,3^{\epsilon}$ & $34,8 \pm 3,3^{\#}$ & $45,8 \pm 3,3^{* * *}$ & $54,2 \pm 2,7^{* *}$ & $71,7 \pm 5,7^{*}$ \\
Média de IMC \pm dp & $23,6 \pm 5,2$ & $23,7 \pm 4,0$ & $24,7 \pm 4,3$ & $24,6 \pm 3,1$ & $27,3 \pm 4,2^{\infty}$ \\
\hline
\end{tabular}

$\mathrm{n}=$ amostra, $\mathrm{m}=$ masculino, $\mathrm{f}=$ feminino, $\mathrm{dp}=$ desvio-padrão, $\mathrm{IMC}=$ índice de massa corpórea. ${ }^{*} \mathrm{p} \leq 0,05$ em relação à média de idade entre todas as faixas etárias; $* * \mathrm{p} \leq 0,05 \mathrm{em}$ relação à média de idade entre as faixas etárias: 20-29, 30-39 e $\geq 60$ anos; $* * * \mathrm{p} \leq 0,05$ em relação à média de idade entre as faixas etárias: 20-29 e $\geq 60$ anos; \#p $\leq 0,05$ em relação à média de idade entre as faixas etárias: 50-59 e $\geq 60$ anos; $€ \mathrm{p} \leq 0,05$ em relação à média de idade entre as faixas etárias: 40-49, 50-59 e $\geq 60$ anos; $\infty$ p $\leq 0,05$ em relação à média de IMC entre as faixas etárias: 20-29 e 30-39 anos.

$\mathrm{Na}$ figura 2, observa-se a comparação das médias das porcentagens das inclinações laterais (assimetria frontal) entre as faixas etárias, na qual há maior lateralização do corpo com o avançar da idade. Os valores são significativos nos grupos $50-59$ e $\geq 60$ anos, indicando que, quanto mais idosa a pessoa, maior o risco de desequilíbrio lateral na postura estática.

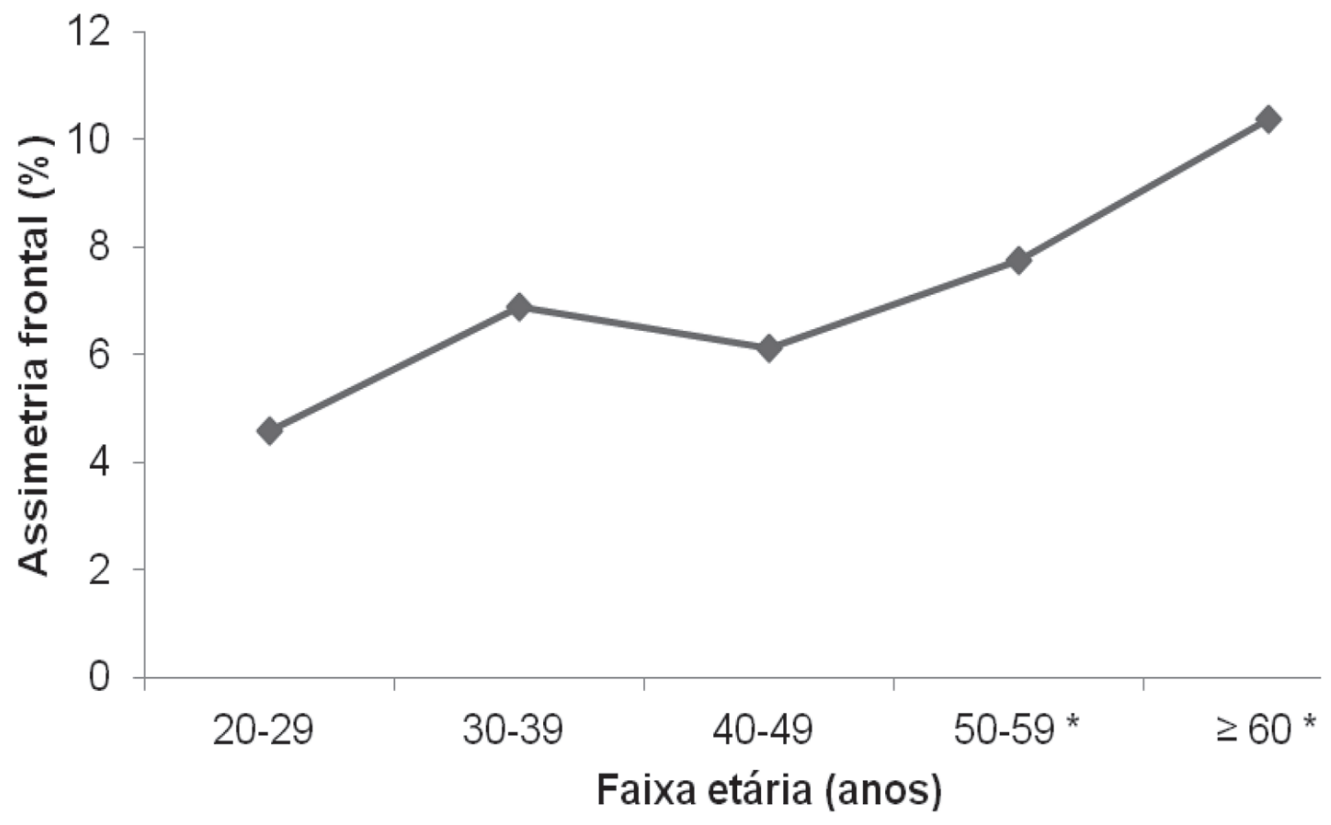

*p $\leq 0,05$ em relação às faixas etárias 20-29 e 40-49 anos

Figura 2 - Média das porcentagens da assimetria frontal separadas por faixas etárias. Marília-SP, 2011.

A figura 3 representa as médias das porcentagens das inclinações ântero-posteriores (assimetria sagital) entre as faixas etárias, com valores significativos no grupo dos $\geq 60$ anos.
Esses dados significam que, acima dessa faixa etária, ocorre maior risco de desequilíbrio ântero-posterior. 


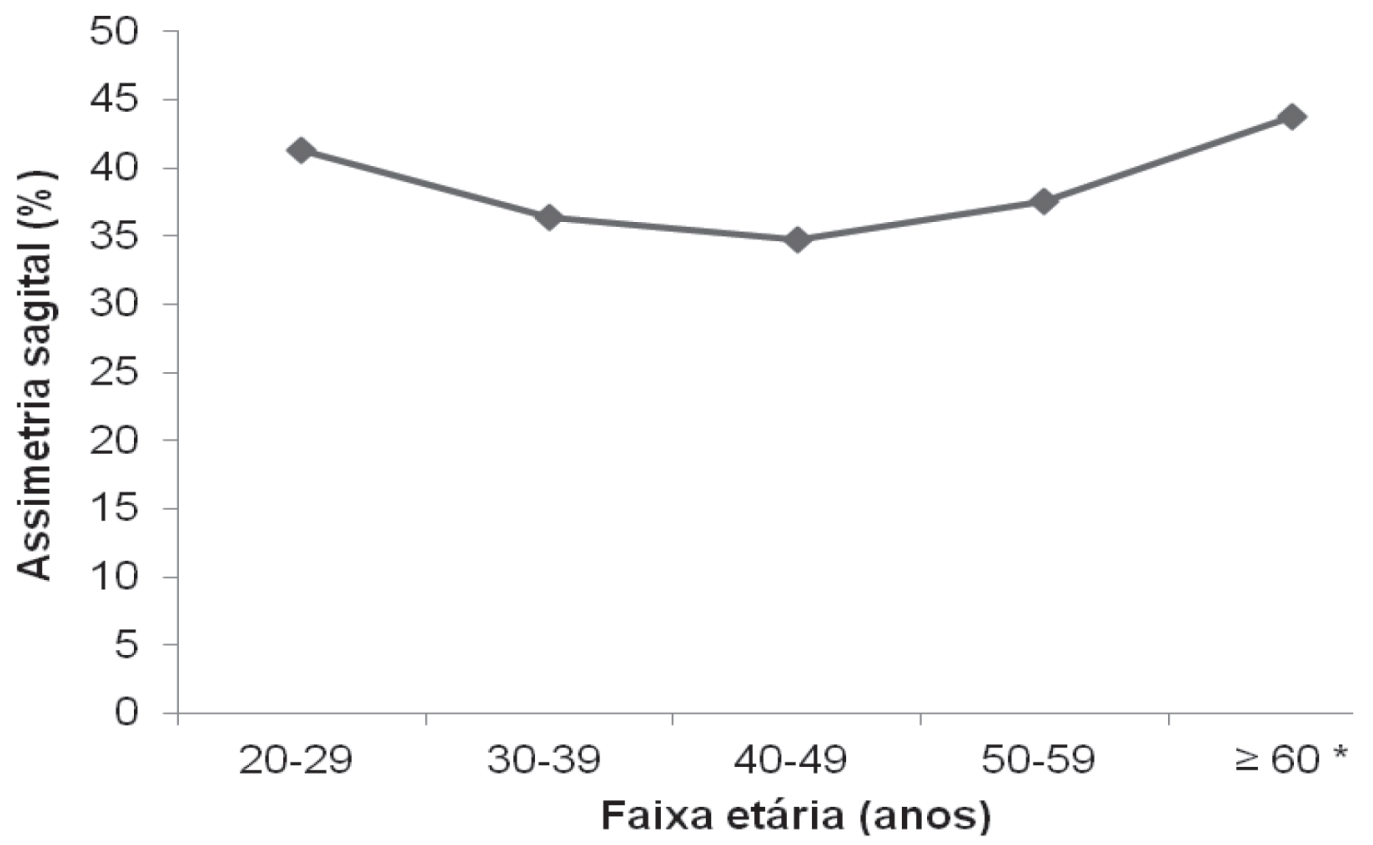

${ }^{*} \mathrm{p} \leq 0,05$ em relação à faixa etária 40-49 anos.

Figura 3 - Média das porcentagens da assimetria sagital separadas por faixas etárias. Marília-SP, 2011.

\section{DISCUSSÃO}

Estudos na literatura apontam que as oscilações posturais aumentam com a idade. ${ }^{14}$ Shaldon ${ }^{15}$ relatou que o controle ideal da oscilação postural é alcançado durante o final da adolescência e mantido até os 60 anos, aproximadamente.

Em 2006, Era et al. ${ }^{16}$ avaliaram o equilíbrio postural por meio de uma plataforma de força em 7.979 indivíduos com 30 anos ou mais de idade e constataram que a deterioração em função do equilíbrio começou em uma idade relativamente jovem e foi acelerada a partir dos 60 anos.

Os resultados do presente estudo apontaram para uma tendência a anteriorização e lateralização do centro de gravidade de acordo com a faixa etária, ou seja, quanto mais idoso, maior o risco de anteriorização e de lateralização do tronco na postura estática. Fonseca \& Scheicher ${ }^{17}$ encontraram correlação entre os escores da escala de equilíbrio de Berg (EEB) e as inclinações lateral e ântero-posterior, sugerindo que quanto maior a projeção anterior (assimetria do plano sagital) e a projeção lateral (assimetria do plano frontal), menores os escores da EEB, indicando aumento do desequilíbrio postural e, portanto, maior risco de cair.

Em seu estudo, Greenspan ${ }^{18}$ apontou que a anteriorização e a lateralização do tronco são posturas que se evidenciam no processo de envelhecimento; relata ainda que são fatores que interferem no equilíbrio, aumentando o risco de quedas. 
Hilliard et al. ${ }^{19}$ relatam que, embora a maioria dos estudos preditores de quedas tenha focalizado no equilíbrio e no desempenho da marcha envolvendo o plano ântero-posterior, há evidências de que os efeitos do envelhecimento sobre o equilíbrio pode ser acentuado no plano látero-lateral. Os resultados encontrados demonstram que há crescente desequilíbrio lateral com o avançar da idade.

Na figura 2, observa-se que dos 20 aos 49 anos houve pouca variação do centro de gravidade na assimetria frontal; o mesmo foi observado na figura 3 para a assimetria lateral em relação à faixa etária dos 40-49 anos. Uma explicação para esse fato é que sujeitos jovens e de meia idade oscilam menos do que indivíduos mais velhos. ${ }^{15}$

Este estudo utilizou uma tecnologia de fácil acesso e manipulação, com download gratuito, instruções autoexplicativas e validado, ${ }^{13}$ para que possa ser aplicado na prática clínica. Portanto, tendo em vista que os declínios no controle postural causados pelo processo de envelhecimento envolvem os planos sagital e frontal, a avaliação postural baseada em imagens possibilita a identificação de posturas irregulares, a prevenção de patologias e a elaboração de intervenções. $^{20}$ Identificar a faixa etária em que as alterações posturais começam a ocorrer pode facilitar a adoção de ações preventivas em relação à queda, um grande problema de saúde pública. ${ }^{8,14,21}$

Uma das limitações do estudo foi o número desigual de participantes em cada grupo e o tamanho amostral. Apesar disso, os resultados fornecem informações importantes a respeito do tema. Faz-se necessário, portanto, realizar outros estudos com número maior de participantes.

\section{CONCLUSÃO}

Com base nas avaliações realizadas, podese afirmar que ocorre aumento das inclinações anteroposterior e lateral com o avançar da idade, sugerindo maior desequilíbrio e, consequentemente, maior risco de quedas. Os resultados podem contribuir para o desenvolvimento de políticas públicas com vistas à prevenção da ocorrência de quedas, incentivando a realização de programas a partir dos 50 anos de idade.

\section{REFERÊNCIAS}

1. Instituto Brasileiro de Geografia e Estatística. Sala de imprensa: canal de comunicação entre o IBGE e os jornalistas [acesso em 20 nov 2011]. Disponível em: http://www.ibge.gov.br/home/presidencia/noticias/ noticia_visualiza.php?id_noticia=1866\&id_pagina=1.

2. Toledo DR, Barela JA. Diferenças sensoriais e motoras entre jovens e idosos: contribuição somatossensorial no controle postural. Rev Bras Fisioter 2010;14(3):267-75.

3. Maki BE, Mcllroy WE. Postural control in the older adult. Clin Geriatr Med 1996;12(4):635-58.

4. Horak FB, Macpherson JM. Postural orientation and equilibrium. In: Rowell LB, Shepard JT, editors. Handbook of physiology: section 12, exercise regulation and integration of multiple systems. New York: Oxford University Press; 1996. p. 255-92.

5. Horak FB. Postural orientation and equilibrium: what do we need to know about neural control of balance to prevent falls? Age Ageing 2006;35(Suppl 2):ii7-11.

6. Wade MG, Lindquist R, Taylor JR, Treat-Jacobson D. Optical flow, spatial orientation, and the control of posture in the elderly. J Gerontol B Psychol Sci Soc Sci 1995;50(1):P51-8.

7. Bierbaum S, Peper A, Arampatzis A. Exercise of mechanisms of dynamic stability improves the stability state after an unexpected gait perturbation in elderly. Age (Dordr) 2013;35(5):190515. [Acesso em 18 set 2011]. In: Epub 2012 Out 10 [Epub ahead of print].

8. Brasil. Ministério da Saúde. Portal da Saúde [acesso em 20 set 2011] Disponível em: http:// portal.saude.gov.br/portal/saude/visualizar_texto. cfm?idtxt $=33674 \&$ janela $=1$.

9. Lajoie Y, Gallagher SP. Predicting falls within the elderly community: comparison of postural sway, reaction time, the Berg balance scale and the Activities-specific Balance Confidence (ABC) scale for comparing fallers and non-fallers. Arch Gerontol Geriatr 2004;38(1):11-26. 
10. Simoceli L, Bittar RMV, Bottino MA, Bento RF. Perfil diagnóstico do idoso portador de desequilíbrio corporal: resultados preliminares. Rev Bras Otorrinolaringol 2003;69(6):772-7.

11. Maciel ACC, Guerra RO. Prevalência e fatores associados ao defícit de equilíbrio em idosos. Rev Bras Cienc Mov 2005;13(1):37-44.

12. Brucki SMD, Nitrini R, Caramelli P, Bertolucci PHF, Okamoto IH. Sugestões para o uso do mini-exame do estado mental no Brasil. Arq Neuro-psiquiatr 2003;61(3b):777-81.

13. Ferreira EAG, Duarte M, Maldonado EP, Burke TN, Marques AP. Postural assessment software (PAS/SAPO): validation and reliability. Clinics 2010;65(7):675-81.

14. Liaw MY, Chen CL, Pei YC, Leong CP, Lau YC. Comparison of the static and dynamic balance performance in young, middle-aged, and elderly healthy people. Chang Gung Med J 2009;32(3):297-304.

15. Sheldon JH. The effect of age on the control of sway. Gerontol Clin (Based) 1963;5:129-38.

16. Era P, Sainio P, Koskinen S, Haavisto P, Vaara M, Aromaa A. Postural balance in a random sample of
7,979 subjects aged 30 years and over. Gerontology 2006;52(4):204-13

17. Fonseca LCS, Scheicher ME. Relação entre projeção do centro de gravidade e equilíbrio em idosos. Ter Man 2012;10(50):440-3.

18. Greenspan SL, Myers ER, Kiel DP, Parker RA, Hayes WC, Resnick NM. Fall direction, bone mineral density, and function: risk factors for hip fracture in frail nursing home elderly. Am J Med 1998;104(6):539-45.

19. Hilliard MJ, Martinez KM, Janssen I, Edwards B, Mille ML, Zhang Y, Rogers MW. Lateral balance factors predict future falls in community-living older adults. Arch Phys Med Rehabil 2008;89(9):1708-13.

20. Arias KC, Readi NG, Vieira TMM, Oliveira LF. Análise comparativa de alinhamentos posturais entre mulheres jovens e idosas. Rio Claro: UNESP; 2007 [acesso em 18 set 2011]. Disponível em: http://www2.rc.unesp.br/eventos/ educacao_fisica/biomecanica2007/upload/146-1-Bposturacbbfinalsemleg.pdf

21. Rogind H, Lykkegaard JJ, Bliddal H, DanneskioldSamsoe B. Postural sway in normal subjects aged 20-70 years. Clin Physiol Funct Imaging. 2003 ;23(3):171-6. 